Document downloaded from:

http://hdl.handle.net/10251/160809

This paper must be cited as:

Herrero Debón, A.; Thome, N. (2020). Sharp partial order and linear autonomous systems. Applied Mathematics and Computation. 366:1-11. https://doi.org/10.1016/j.amc.2019.124736

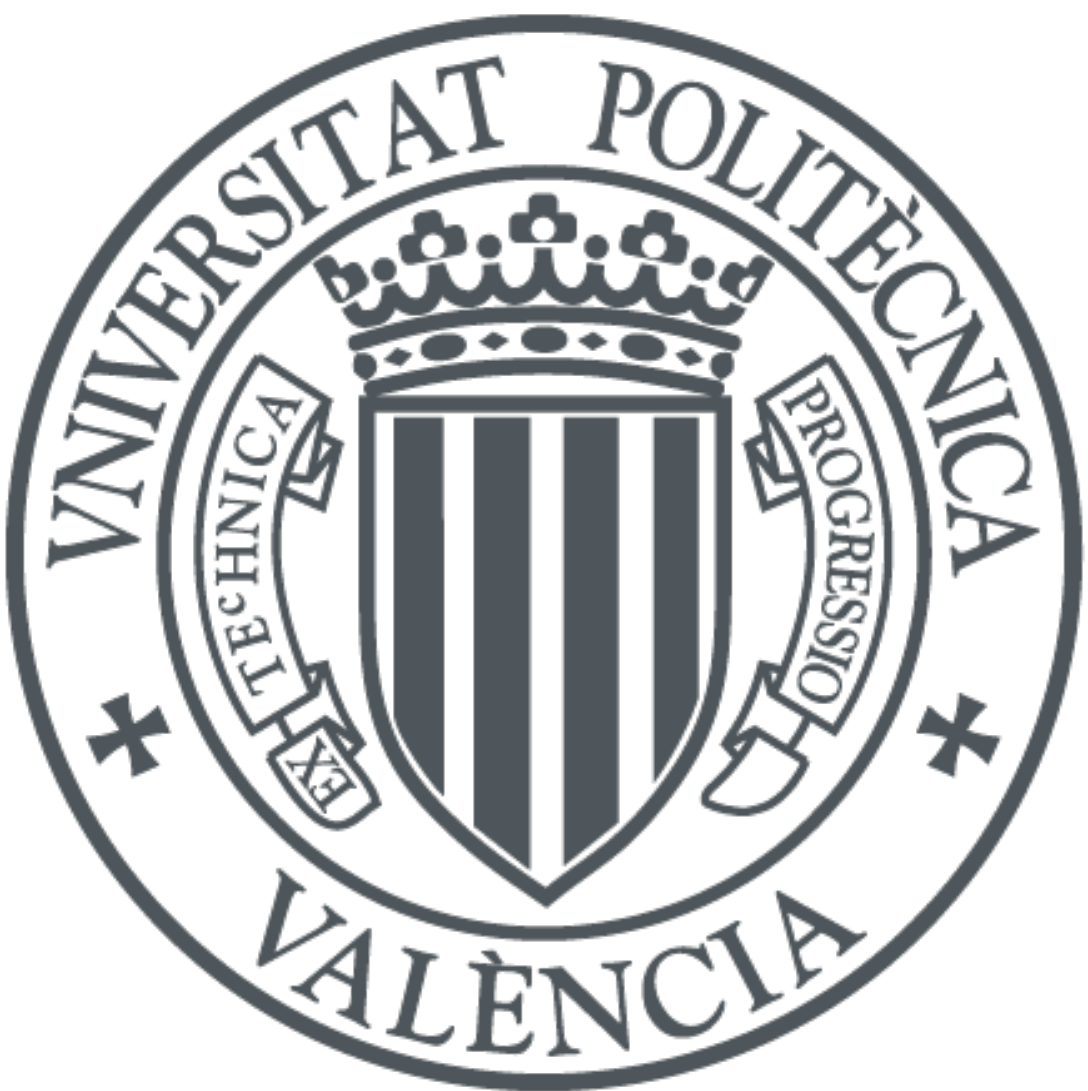

The final publication is available at

https://doi.org/10.1016/j.amc.2019.124736

Copyright Elsevier

Additional Information 


\title{
Sharp partial order and linear autonomous systems
}

\author{
A. Herrero and N. Thome ${ }^{1}$ \\ Instituto Universitario de Matemática Multidisciplinar \\ Universitat Politècnica de València \\ Camino de Vera, 14. 46022 Valencia. Spain
}

\begin{abstract}
This paper deals with autonomous linear systems and the sharp partial order. Given an autonomous linear system, we find another system, which is related to the first one by means of the sharp partial order. This relation can be interpreted in different ways: as a perturbation or as a projection of the initial system. Both points of view allow us to work with a new system with some previously selected behaviour. The solutions of the two systems are related via a matrix that gives the gap between them. We design some algorithms and analize their performance with numerical examples.
\end{abstract}

Keywords: Generalized inverses, sharp partial order, linear systems 2010 MSC: 15A09, 06A06

\section{Introduction}

Autonomous linear systems are a very important class of (ordinary) differential equations and difference equations. They are used as mathematical models representing real processes in order to analyze their behaviour. It is well known that the investigation of the crucial characteristic of a system of being stable requires some background of linear algebra and matrix theory [15]. Another feature of linear autonomous systems is their behaviour under certain perturbations [13. Under these circumstances, the new system differs by a small amount from the original autonomous system and is called the perturbed system. The important question to answer in this case is how close is the solution of a perturbed system to the solution of the original one as J. Cronin pointed out in [6. Some results related to perturbations of linear systems and matrices were given by J.Y. Vélez-Cerrada, J. Robles, N. Castro-González, and Y. Wei and they can be found in [20, 21].

On the other hand, in the last decades matrix partial orders are receiving an increasing attention because of their usefulness in different areas. The excellent monograph published by S.K. Mitra, P. Bhimasankaram, S.B. Malik

\footnotetext{
${ }^{1}$ Corresponding author. Email: njthome@mat.upv.es
}

Preprint submitted to Journal of Applied Mathematics and Computation September 9, 2019 
(see [17]) gave rise to a great impulse to the research in this topic. For instance, mathematics morphology is a powerful tool to study geometrical structures with applications to image processing as J. Angulo shows in [1]. Moreover, in Statistics, two Gauss-Markov linear systems can be compared in the sense that one of them is as good as the other provided that some covariance matrices are related under a matrix partial order as G.P.H. Styan stated in [19]. Some results related to partial orders were given by D.S. Cvetković-Ilić, D. Mosić, and Y. Wei and can be found in [7, 16].

The novelty of our contribution in this paper consists in the combination of these two areas (autonomous linear systems and matrix partial orders) producing a new outlook at autonomous systems. For that, perturbation and projection matrices will be considered in order to relate the solutions of two special autonomous linear systems with coefficient matrices ordered under the sharp partial order.

The main aim of this paper is to present two approaches for the comparison of two autonomous systems ordered under the sharp partial order. A similar relationship to that considered in this paper was given in [5] although solution vectors in both systems could not be compared. However, using sharp partial order we can carry out this comparison, which shows that the consideration of sharp partial order (instead of minus partial order) represents an important advantage since much more information can be obtained.

Throughout this paper we will use the following notation. Let $A$ be a real square matrix. The spectrum of $A$ is defined as the set of all its eigenvalues and denoted by $\sigma(A)$. The spectral radius of $A$ is defined as $\rho(A)=\max \{|\lambda|: \lambda \in$ $\sigma(A)\}$.

A square matrix $A$ is said to be an index 1 matrix if $A$ and $A^{2}$ have the same rank. We recall that for a given $n \times n$ matrix $A$ of index 1 , the symbol $A^{\#}$ denotes the group inverse of $A$ which satisfies $A A^{\#} A=A, A^{\#} A A^{\#}=A^{\#}$, and $A A^{\#}=A^{\#} A$ (see [3]). The index 1 condition of $A$ ensures the existence of its group inverse.

For two index 1 given matrices $A_{1}, A_{2} \in \mathbb{R}^{n \times n}$, it is well known that $A_{2}$ is a successor of $A_{1}$ under the sharp partial order if $A_{1} A_{1}^{\#}=A_{2} A_{1}^{\#}$ and $A_{1}^{\#} A_{1}=$ $A_{1}^{\#} A_{2}$. This binary relation will be denoted by $A_{1} \stackrel{\#}{\leq} A_{2}$ (see [17]).

Given a matrix $A \in \mathbb{R}^{n \times n}$, we will need the Frobenius norm defined by $\|A\|_{F}=\operatorname{trace}\left(A^{T} A\right)$ where $A^{T}$ denotes the transpose of $A$, and the $P$-matrix norm defined by $\|A\|_{P}=\left\|P^{-1} A P\right\|_{F}$. by

The representation of a discrete-time (linear) autonomous system is given

$$
x(k+1)=A x(k), \quad k=0,1, \ldots,
$$

where $x(k) \in \mathbb{R}^{n}, k=0,1, \ldots$, and $A \in \mathbb{R}^{n \times n}$ is the state coefficient matrix. It is well known that the solution of system (1) is given by the expression

$$
x(k)=A^{k} x(0),
$$

where $x(0)$ is an admissible initial condition. 
An important concept related to autonomous systems is Stability. It is said that system (1) is asymptotically stable if $\rho(A)<1$.

It is well known that important applications of systems like (1) appear in Markov chains [8] as well as in Leslie models [4, among others.

This paper is organized as follows. Section 2 introduces the concept of ordered autonomous linear system under the sharp partial order. This binary relation can be interpreted as a perturbation of the predecessor system. Unlike the comparison done under the minus partial order used in [5], the important fact in this paper is that the solutions of both systems are related by means of the perturbation matrix. Moreover, the gap between both solutions is upper bounded in terms of the Frobenius norm of the perturbation matrix. In addition, an algorithm has been designed and a numerical example shows its performance. Section 3 gives a different approach where the predecessor matrix is obtained as a projection of the successor matrix. Even more, the same projection relation is valid for the solution vectors. The structure of Section 3 continues similarly to that of Section 2 by providing an upper bound, an algorithm, and numerical examples to illustrate this second approach. Finally, in Section 4 we derive a new tool by introducing a third point of view for understanding two related systems where all the projectors we can choose are explicitly found.

\section{Partially ordered perturbed system}

In this section we are going to consider autonomous systems and introduce an order relation between two given systems in order to compare them.

For that, throughout this section, we will consider the following systems:

$$
x(k+1)=A_{1} x(k), \quad \text { and } \quad \bar{x}(k+1)=A_{2} \bar{x}(k), \quad k=0,1, \ldots
$$

where $A_{1}, A_{2} \in \mathbb{R}^{n \times n}$ have index 1 .

We remark that (singular) diagonalizable matrices give rise to a wide class of matrices that may appear in (3) because all of them have index 1 . Since the set of diagonalizable matrices is dense in the set of all matrices [9], we conclude that it is quite common in practice to find situations where index 1 matrices do appear. For instance, in Biology, autonomous systems are used to represent the population growth of a certain species. The matrix appearing in this kind of models is known as Leslie matrix and has the form [11, 12.

$$
L=\left[\begin{array}{ccccc}
a_{1} & a_{2} & \cdots & a_{n-1} & a_{n} \\
b_{1} & 0 & \cdots & 0 & 0 \\
0 & b_{2} & \cdots & 0 & 0 \\
\vdots & \vdots & \vdots & \vdots & \vdots \\
0 & 0 & \cdots & b_{n-1} & 0
\end{array}\right]
$$

where $a_{i} \geq 0, i=1, \ldots, n$ and $0<b_{i} \leq 1, i=1, \ldots, n-1$, depend on the 
natality, mortality, and age of the population. For instance,

$$
L=\left[\begin{array}{ccc}
2 & 0.3 & 0 \\
0.8 & 0 & 0 \\
0 & 0.1 & 0
\end{array}\right]
$$

is a Leslie matrix, it has index 1 and is diagonalizable.

Definition 1. The autonomous linear systems given by (3) are said to be ordered under the sharp partial order if $A_{1} \stackrel{\#}{\leq} A_{2}$.

Now, we establish the relationship between the solutions of two given autonomous systems related by the sharp partial order. To do that, we recall the following characterization of the sharp partial order.

Proposition 1. [2] Let $A_{1} \in \mathbb{R}^{n \times n}$ be a matrix written as

$$
A_{1}=P\left[\begin{array}{lll}
C & & \\
& O & \\
& & O
\end{array}\right] P^{-1}
$$

with $C \in \mathbb{R}^{r \times r}$ and $P \in \mathbb{R}^{n \times n}$ being nonsingular. Then, the following assertions are equivalent:

(a) There exists $A_{2} \in \mathbb{R}^{n \times n}$ such that $A_{1} \stackrel{\#}{\leq} A_{2}$.

(b) There exists a nonsingular matrix $Y \in \mathbb{R}^{\ell \times \ell}$ such that

$$
A_{2}=P\left[\begin{array}{lll}
C & & \\
& Y & \\
& & O
\end{array}\right] P^{-1} .
$$

The expression (4) is known as the core-nilpotent decomposition of $A_{1}[3$. The missing blocks in the middle matrix in (4) are null matrices of adequate sizes. This notation will be used in what follows.

This characterization allows us to write the following result.

Theorem 2. Let $A_{1}, A_{2} \in \mathbb{R}^{n \times n}$ be the state matrices of two autonomous systems given by (3) ordered under the sharp partial order. Then, there exists a matrix $\Gamma \in \mathbb{R}^{n \times n}$ such that the solutions of both systems are related by

$$
\bar{x}(k)=x(k)+\Gamma^{k} x(0)
$$

provided that both initial conditions are equal, that is $\bar{x}(0)=x(0)$.

Proof. Assume that $A_{1} \stackrel{\#}{\leq} A_{2}$. By Proposition 1 1 , there exists a nonsingular matrix $P \in \mathbb{R}^{n \times n}$ such that

$$
A_{2}=A_{1}+P\left[\begin{array}{ccc}
O & & \\
& Y & \\
& & O
\end{array}\right] P^{-1} .
$$


Set

$$
\Gamma=P\left[\begin{array}{ccc}
O & & \\
& Y & \\
& & O
\end{array}\right] P^{-1} .
$$

Since $A_{1} \Gamma=\Gamma A_{1}=O$, Newton's binomial formula gives

$$
A_{2}^{k}=\sum_{j=0}^{k}\left(\begin{array}{c}
k \\
j
\end{array}\right) A_{1}^{j} \Gamma^{k-j}=A_{1}^{k}+\Gamma^{k} .
$$

Then, the solution of the second system is

$$
\bar{x}(k)=A_{2}^{k} \bar{x}(0)=\left(A_{1}^{k}+\Gamma^{k}\right) x(0)=x(k)+\Gamma^{k} x(0) .
$$

Remark 1. An interesting observation is that the vector $\bar{x}(k)$ in solution (6) can be interpreted as a perturbation of the vector $x(k)$. Therefore, the solution of the successor system is obtained as a perturbation of the solution of the predecessor system under the sharp partial order.

The previous result allows us to give an upper bound for the difference between both solutions.

Corollary 3. Let $x(k)$ and $\bar{x}(k)$ be the solutions of the systems given in (3). If both systems are ordered under the sharp partial order then

$$
\|\bar{x}(k)-x(k)\| \leq\|Y\|_{F}^{k}\|x(0)\|,
$$

where the vector norm $\|\cdot\|$ is a compatible norm with the P-matrix norm.

Proof. By Theorem 2, we have $\bar{x}(k)=x(k)+\Gamma^{k} x(0)$. For a vector norm $\|$. compatible with the $P$-matrix norm, we can write

$$
\|\bar{x}(k)-x(k)\|=\left\|\Gamma^{k} x(0)\right\| \leq\left\|\Gamma^{k}\right\|_{P}\|x(0)\| \leq\|\Gamma\|_{P}^{k}\|x(0)\| .
$$

By using the relation (8), we get

$$
\|\Gamma\|_{P}=\left\|P^{-1} \Gamma P\right\|_{F}=\left\|\left[\begin{array}{ccc}
O & & \\
& Y & \\
& & O
\end{array}\right]\right\|_{F}=\|Y\|_{F} .
$$

Hence, the result holds.

Notice that if the system given by $A_{2}$ is constructed with a "small" matrix $Y$, we can assure that the difference between the two solutions is also small and an upper bound for this difference is given in terms of the norm of $Y$. 
Remark 2. We can observe that the system defined by $A_{2}$ can be seen as a perturbation of the system given by $A_{1}$. In this case, Corollary 3 guarantees that the solution $\bar{x}(k)$ is as close to the solution $x(k)$ as the magnitude of the perturbation matrix $Y$ is. For instance, if we choose $Y=\operatorname{diag}\left(\epsilon_{1}, \ldots, \epsilon_{\ell}\right)$ with $0<\epsilon_{i}<<1$ for $i=1, \ldots, \ell$, we obtain that $\sigma\left(A_{2}\right)=\sigma(C) \cup \sigma(Y)$. This fact implies that the stability of the system defined by $A_{1}$ is preserved in the perturbed system given by $A_{2}$. However, starting from a stable system given by $A_{1}$, if we perturb it with a unstable $Y$, the obtained system given by $A_{2}$ is always unstable.

\subsection{Algorithm and example}

For a given matrix $A_{1}$, the algorithm below constructs the solution of the systems $x(k+1)=A_{1} x(k)$ and $\bar{x}(k+1)=A_{2} \bar{x}(k)$ satisfying that $A_{1}$ is below $A_{2}$ under the sharp partial order.

Algorithm 1. Inputs: The matrix $A_{1}$ of index at most 1, the initial condition $x_{0}$, and the nonzero perturbation numbers $\epsilon_{1}, \ldots, \epsilon_{\ell}$.

Outputs: The matrix $A_{2}$ and the solutions $x(k)$ and $\bar{x}(k)$.

Step 1. Compute the core-nilpotent decomposition of $A_{1}$, i.e.,

$$
A_{1}=P\left[\begin{array}{ccc}
C & & \\
& O & \\
& & O
\end{array}\right] P^{-1} .
$$

Step 2. Select $Y=\operatorname{diag}\left(\epsilon_{1}, \ldots, \epsilon_{\ell}\right)$.

Step 3. Construct $A_{2}$ as in (5).

Step 4. Compute the perturbation matrix $\Gamma=P\left[\begin{array}{lll}O & & \\ & Y & \\ & & O\end{array}\right] P^{-1}$.

Step 5. The solutions are: $x(k)=A_{1}^{k} x_{0}$ and $\bar{x}(k)=x(k)+\Gamma^{k} x_{0}$.

Notice that in the algorithm we have $\|Y\|_{F}=\sum_{i=1}^{\ell} \epsilon_{i}^{2}$, which allows us to obtain the upper bound $\|Y\|_{F}^{k}\|x(0)\|$ of the gap $\|\bar{x}(k)-x(k)\|$.

This algorithm and the others appearing in the following sections can be easily implemented on a computer. We have used the Wolfram Mathematica 11.3 (2018) package. For each algorithm we present some numerical examples in order to show the performance and demonstrate the applicability.

Example 4. Consider the autonomous system $x(k+1)=A_{1} x(k)$ given by the matrix

$$
A_{1}=\left[\begin{array}{rrrrrr}
0.1739 & -0.0607 & -0.0662 & 0.0763 & 0.0596 & -0.0400 \\
0.4338 & -0.0725 & -0.1948 & 0.2847 & 0.1164 & -0.0720 \\
-0.8839 & 0.3844 & 0.3080 & -0.2966 & -0.3343 & 0.2302 \\
-0.0239 & 0.1583 & -0.0471 & 0.1691 & -0.0697 & 0.0584 \\
0.1986 & -0.1166 & -0.0579 & 0.0304 & 0.0875 & -0.0624 \\
-0.5959 & 0.2936 & 0.1947 & -0.1587 & -0.2395 & 0.1674
\end{array}\right]
$$


This matrix has index 1 and can be decomposed as in (4) where

$$
P=\left[\begin{array}{rrrrrr}
0.5377 & -0.4336 & 0.7254 & 1.4090 & 0.4889 & 0.8884 \\
1.8339 & 0.3426 & -0.0631 & 1.4172 & 1.0347 & -1.1471 \\
-2.2588 & 3.5784 & 0.7147 & 0.6715 & 0.7269 & -1.0689 \\
0.8622 & 2.7694 & -0.2050 & -1.2075 & -0.3034 & -0.8095 \\
0.3188 & -1.3499 & -0.1241 & 0.7172 & 0.2939 & -2.9443 \\
-1.3077 & 3.0349 & 1.4897 & 1.6302 & -0.7873 & 1.4384
\end{array}\right]
$$

and

$$
C=\left[\begin{array}{rr}
0.5000 & -0.3333 \\
0 & 0.3333
\end{array}\right] .
$$

If we perturb the previous system considering $A_{2}$ as in (5) where

$$
Y=\left[\begin{array}{rr}
0.5000 & 0 \\
0 & 0.5000
\end{array}\right]
$$

we get the matrix

$$
A_{2}=\left[\begin{array}{rrrrrr}
0.3748 & -0.0548 & -0.1024 & 0.0447 & 0.2241 & 0.1326 \\
-0.2717 & 0.4732 & -0.2945 & -0.1694 & 0.0093 & 0.2499 \\
-0.3656 & 0.1359 & 0.3203 & -0.1150 & -0.1290 & 0.2435 \\
0.2810 & -0.1305 & 0.0172 & 0.4182 & -0.0674 & -0.1675 \\
-0.2640 & 0.2224 & -0.1157 & -0.2485 & 0.0016 & 0.1178 \\
0.3813 & -0.1425 & 0.2049 & 0.1514 & 0.1744 & 0.2454
\end{array}\right]
$$

which satisfies $A_{1} \stackrel{\#}{\leq} A_{2}$. This matrix defines a new system $\bar{x}(k+1)=A_{2} \bar{x}(k)$.

Choosing the above matrix $Y$, Corollary 3 ensures that the solutions of both systems will be as close as indicated by $\|Y\|_{F}=0.7071$.

Taking the initial conditions $x(0)=\bar{x}(0)=\left[\begin{array}{llllll}1 & 1 & 1 & 1 & 1 & 1\end{array}\right]^{T}$, by Theorem 2 their solutions are related by means of the expression (6) as it is shown in Figure 1. In this figure, we can observe the evolution of the 15th first iterations of the solutions $x_{i}(k)$ and $\bar{x}_{i}(k)$. Obviously, the bigger the value of $k$ is, the closer the solutions $x_{i}(k)$ and $\bar{x}_{i}(k)$ are.

\section{Partially ordered projected system}

In this section, we point out another interpretation for two ordered systems under the sharp partial order. In order to do this, let us consider the HartwigSpindelböck decomposition of a given nonzero matrix $A_{2}$ of rank $r$ [10. There exist an orthogonal matrix $U \in \mathbb{R}^{n \times n}$, a diagonal positive definite matrix $\Sigma \in$ $\mathbb{R}^{r \times r}$, and matrices $K \in \mathbb{R}^{r \times r}$ and $L \in \mathbb{R}^{r \times(n-r)}$ such that

$$
A_{2}=U\left[\begin{array}{cc}
\Sigma K & \Sigma L \\
O & O
\end{array}\right] U^{T}
$$

with $K K^{T}+L L^{T}=I_{r}$. If $K$ is nonsingular then $A_{2}$ has index at most 1 .

We quote the following result. 
Proposition 5. 14] Let $A_{2} \in \mathbb{R}^{n \times n}$ be a nonzero matrix written as in (10) with $K$ nonsingular. Then, the following assertions are equivalent

(a) There exists $A_{1} \in \mathbb{R}^{n \times n}$ having index 1 such that $A_{1} \stackrel{\#}{\leq} A_{2}$.

(b) There exists an idempotent matrix $T \in \mathbb{R}^{r \times r}$ that commutes with $\Sigma K$ such that

$$
A_{1}=U\left[\begin{array}{cc}
T \Sigma K & T \Sigma L \\
O & O
\end{array}\right] U^{T} .
$$

Theorem 6. Let $A_{1}, A_{2} \in \mathbb{R}^{n \times n}$ be the state matrices of two autonomous systems given by (3) ordered under the sharp partial order. Then, there exists a matrix $\widetilde{\Gamma} \in \mathbb{R}^{n \times n}$ such that the solutions of both systems are related by

$$
x(k)=\widetilde{\Gamma} \bar{x}(k)
$$

provided that both initial conditions are equal, that is $\bar{x}(0)=x(0)$.

Proof. Assume that $A_{1} \stackrel{\#}{\leq} A_{2}$. Consider the Hartwig-Spindelböck decomposition of $A_{2}$ as in (10). By Proposition 5 there exists an idempotent matrix $T \in \mathbb{R}^{r \times r}$ that commutes with $\Sigma K$ such that $A_{1}$ can be expressed as in (11). It is easy to see that

$$
A_{1}^{k}=U\left[\begin{array}{cc}
(T \Sigma K)^{k} & (T \Sigma K)^{k-1} \Sigma L \\
O & O
\end{array}\right] U^{T}
$$

and

$$
A_{2}^{k}=U\left[\begin{array}{cc}
(\Sigma K)^{k} & (\Sigma K)^{k-1} \Sigma L \\
O & O
\end{array}\right] U^{T}
$$

Then, the solution of the first system is

$$
x(k)=A_{1}^{k} x(0)=U\left[\begin{array}{cc}
T & O \\
O & I_{n-r}
\end{array}\right] U^{T} U\left[\begin{array}{cc}
(\Sigma K)^{k} & (\Sigma K)^{k-1} \Sigma L \\
O & O
\end{array}\right] U^{T} x(0) .
$$

Setting

$$
\widetilde{\Gamma}=U\left[\begin{array}{cc}
T & O \\
O & I_{n-r}
\end{array}\right] U^{T}
$$

we obtain $x(k)=\widetilde{\Gamma} \bar{x}(k)$.

From (??) and 13 we notice that $A_{1}$ can be written as a projection of $A_{2}$, namely $A_{1}=\widetilde{\Gamma} A_{2}$.

Remark 3. An interesting observation is that expression (12) represents the vector $x(k)$ as a projection of the vector $\bar{x}(k)$. Therefore, the solution of the predecessor system is obtained as a projection of the solution of the successor system under the sharp partial order. That is, unlike Theorem 2 (where system defined by $A_{2}$ was constructed as a perturbation of system defined by $A_{1}$ ), in the current approach, Theorem 6 allows us to construct the system defined by $A_{1}$ from the system given by $A_{2}$ by means of an adequate projection. 
This result allows us to obtain the distance between both solutions.

Corollary 7. Let $x(k)$ and $\bar{x}(k)$ be the solutions of the systems given in (3). If both systems are ordered under the sharp partial order then

$$
\|x(k)-\bar{x}(k)\| \leq\|T-I\|_{F}\|\Sigma\|_{F}^{k}\|x(0)\|
$$

where $I$ represents the identity matrix of suitable size and $x(0)$ is an admissible initial condition.

Proof. By Theorem 6, we have $x(k)=\widetilde{\Gamma} \bar{x}(k)$. For a vector norm $\|$.$\| compat-$ ible with the Frobenius matrix norm, we can write

$$
\|x(k)-\bar{x}(k)\|=\|(\widetilde{\Gamma}-I) \bar{x}(k)\| \leq\|\widetilde{\Gamma}-I\|_{F}\|\bar{x}(k)\|=\|T-I\|_{F}\|\bar{x}(k)\|,
$$

where we have used the expression $\sqrt{13}$ of the matrix $\widetilde{\Gamma}$. Now, taking into account that $\bar{x}(k)=A_{2}^{k} x(0)$ for an admissible initial condition $x(0)$, we have that

$$
\|x(k)-\bar{x}(k)\| \leq\|T-I\|_{F}\left\|A_{2}^{k} x(0)\right\| \leq\|T-I\|_{F}\left\|A_{2}\right\|_{F}^{k}\|x(0)\| .
$$

By using the definition of the Frobenius norm and the decomposition of the matrix $A_{2}$ given in 10 we have that

$$
\begin{aligned}
\left\|A_{2}\right\|_{F}^{2} & =\operatorname{trace}\left(A_{2} A_{2}^{T}\right)=\operatorname{trace}\left(\left[\begin{array}{cc}
\Sigma K & \Sigma L \\
O & O
\end{array}\right]\left[\begin{array}{cc}
K^{T} \Sigma & O \\
L^{T} \Sigma & O
\end{array}\right]\right) \\
& =\operatorname{trace}\left(\left[\begin{array}{cc}
\Sigma\left(K K^{T}+L L^{T}\right) \Sigma & O \\
O & O
\end{array}\right]\right)=\|\Sigma\|_{F}^{2}
\end{aligned}
$$

Hence, the result holds.

Remark 4. Corollary 7 guarantees that the solution $x(k)$ is as close to the solution $\bar{x}(k)$ as the magnitude of the singular value matrix $\Sigma$ is; if $\|\Sigma\|_{F}<1$ then $x(k)$ and $\bar{x}(k)$ will be close for large values of $k$. Table 1 shows all the possible situations that we can have when asymptotic stability is compared with the singular values of the state matrix. Obviously, the interesting cases are those for which matrix $A_{2}$ is singular, the remaining cases are ommited. The property $\left|\operatorname{det}\left(\mathrm{A}_{2}\right)\right|=\prod_{i=1}^{n}\left|\lambda_{i}\right|=\prod_{i=1}^{n} \sigma_{i}$ is used to complete Table 1. For example, the first row in Table 1 means that the matrix $A_{2}$ in Example 9 satisfies $\rho\left(A_{2}\right)<1$, the system given by $A_{2}$ is asymptotically stable, all the eigenvalues of $A_{2}$ have modulus less than 1 , and the sequence $\|\Sigma\|_{F}^{k}$ tends to zero as $k$ tends to infinity. A similar interpretation is valid for the other rows. Note that Examples 9 and 10 mentioned in the Table are located after it. Moreover, while the symbol $\exists$ means it exists, the symbol $\nexists$ means it does not exist. 
Proposition 8. Let $A_{1}, A_{2} \in \mathbb{R}^{n \times n}$ be the state matrices of two autonomous systems given by (3) ordered under the sharp partial order. Then, the stability of the system given by $A_{2}$ implies the stability of the system given by $A_{1}$. Moreover, if $A_{1}$ and $A_{2}$ are written as in (11) and (10), respectively, then

$$
\sigma(T \Sigma K) \subseteq \sigma(\Sigma K) .
$$

Proof. Since $A_{1} \stackrel{\#}{\leq} A_{2}$, by Proposition 1 we can assure that $\sigma\left(A_{1}\right) \subseteq \sigma\left(A_{2}\right)$. Thus, the stability property follows directly. Now, assuming that $A_{1}$ and $A_{2}$ are respectively written as in 11 and $(10)$, we have $\sigma\left(A_{1}\right)=\sigma(T \Sigma K)$ and $\sigma\left(A_{2}\right)=\sigma(\Sigma K)$. This ends the proof.

An interesting observation derives from this proposition. It allows us to ensure that the stability of the system defined by $A_{1}$ is independent of the idempotent matrix $T$.

\subsection{Algorithm and example}

For a given matrix $A_{2}$, the algorithm below constructs the solution of the systems $\bar{x}(k+1)=A_{2} \bar{x}(k)$ and $x(k+1)=A_{1} x(k)$ satisfying that $A_{1}$ is below $A_{2}$ under the sharp partial order.

Algorithm 2. Inputs: The matrix $A_{2}$ of index at most 1 and the initial condition $x_{0}$.

Outputs: The matrix $A_{1}$ and the solutions $\bar{x}(k)$ and $x(k)$.

Step 1. Compute the singular value decomposition of $A_{2}$, that is $A_{2}=U S V^{T}$ and $r=\operatorname{rank}\left(A_{2}\right)$.

Step 2. Assign to $\Sigma$ the first $r$ rows and the first $r$ columns of $S$.

Step 3. Compute $M=S V^{T} U$.

Step 4. Assign to $\widetilde{M}$ the first $r$ rows and the first $r$ columns of $M$.

Step 5. Compute $R=\Sigma^{-1} \widetilde{M}$.

Step 6. Assign to $K$ the first $r$ rows and the first $r$ columns of $R$.

Step 7. Assign to $L$ the first $r$ rows and the last $n-r$ columns of $R$.

Step 8. Find a matrix $T$ such that $\Sigma K T=T \Sigma K$ and $T^{2}=T$.

Step 9. Construct

$$
A_{1}=U\left[\begin{array}{cc}
T \Sigma K & T \Sigma L \\
O & O
\end{array}\right] U^{T} \quad \text { and } \quad \widetilde{\Gamma}=U\left[\begin{array}{cc}
T & O \\
O & I
\end{array}\right] U^{T} .
$$

Step 10. The solutions are: $\bar{x}(k)=A_{2}^{k} x_{0}$ and $x(k)=\widetilde{\Gamma} \bar{x}(k)$. 
Example 9. Consider the autonomous system $\bar{x}(k+1)=A_{2} \bar{x}(k)$ given by the matrix

$$
A_{2}=\left[\begin{array}{rrrrrr}
0.1825 & 0.0776 & -0.1392 & -0.0791 & 0.1889 & 0.1878 \\
0.1054 & 0.2135 & -0.2223 & 0.0736 & 0.0783 & 0.1416 \\
-0.1096 & -0.0404 & 0.3692 & 0.0499 & -0.0821 & 0.1700 \\
-0.0265 & 0.0813 & -0.0417 & 0.2201 & -0.1237 & -0.0791 \\
0.0225 & 0.0251 & -0.0609 & -0.0639 & 0.0540 & 0.0355 \\
0.1198 & 0.0376 & 0.1548 & -0.0170 & 0.1265 & 0.3205
\end{array}\right]
$$

Following the steps in the Algorithm we have that

$$
\Sigma=\left[\begin{array}{cccc}
0.5784 & 0 & 0 & 0 \\
0 & 0.5255 & 0 & 0 \\
0 & 0 & 0.3085 & 0 \\
0 & 0 & 0 & 0.0 .0819
\end{array}\right]
$$

and we can choose the matrix $T$ as

$$
T=\left[\begin{array}{rrrr}
0.5183 & 0.1327 & 0.0672 & 0.6801 \\
1.5347 & 0.6353 & -1.0228 & 0.1730 \\
0.2380 & -0.0320 & 0.4990 & 1.0177 \\
0.0442 & -0.0268 & 0.1978 & 0.3475
\end{array}\right]
$$

such that $A_{1}$ is

$$
A_{1}=\left[\begin{array}{rrrrrr}
0.0597 & -0.0225 & -0.0867 & 0.0702 & 0.0404 & 0.0611 \\
0.0711 & -0.0270 & -0.1448 & 0.0167 & 0.1921 & 0.0437 \\
0.1184 & -0.0441 & -0.0694 & 0.3043 & -0.2750 & 0.1931 \\
0.0935 & -0.0354 & -0.1763 & 0.0446 & 0.2037 & 0.0673 \\
-0.0390 & 0.0147 & 0.0525 & -0.0524 & -0.0122 & -0.0428 \\
0.1833 & -0.0687 & -0.1864 & 0.3441 & -0.1522 & 0.2437
\end{array}\right]
$$

and

$$
\widetilde{\Gamma}=\left[\begin{array}{rrrrrr}
0.5828 & -0.1593 & 0.2395 & 0.3979 & -0.1762 & -0.1974 \\
0.3924 & 0.0523 & -0.6095 & 0.2635 & -0.4731 & 0.3986 \\
-0.5095 & -0.2376 & 1.2773 & 0.5109 & -0.2402 & -0.2327 \\
0.6286 & -0.1836 & -0.5091 & 0.6467 & 0.0189 & 0.3796 \\
-0.0308 & -0.1939 & -0.0461 & 0.1352 & 0.8810 & 0.0208 \\
-0.8719 & -0.1909 & 0.5502 & 0.7490 & -0.2855 & 0.5600
\end{array}\right] .
$$

Then, the magnitude of the difference between the solutions computed by the Algorithm is indicated in Table 2.

Example 10. Consider the autonomous system $\bar{x}(k+1)=A_{2} \bar{x}(k)$ given by the matrix $A_{2}$ obtained in Example 4. Following the steps in the Algorithm we get

$$
\Sigma=\left[\begin{array}{cccc}
1.0819 & 0 & 0 & 0 \\
0 & 0.5784 & 0 & 0 \\
0 & 0 & 0.5256 & 0 \\
0 & 0 & 0 & 0.3085
\end{array}\right]
$$


and we can choose the matrix $T$ as

$$
T=\left[\begin{array}{rrrr}
-0.1860 & -0.1428 & 0.0821 & 0.2657 \\
0.6177 & 0.5894 & -0.3608 & -0.0162 \\
-1.0127 & -0.9133 & 0.5509 & 0.4261 \\
-0.1855 & -0.0384 & 0.0021 & 1.0455
\end{array}\right]
$$

such that $A_{1}$ is exactly the matrix given in Example 4. In this case,

$$
\widetilde{\Gamma}=\left[\begin{array}{rrrrrr}
0.6351 & -0.1026 & 0.0854 & 0.1837 & -0.0565 & -0.3339 \\
0.1969 & 0.3503 & -0.4963 & 0.3695 & -0.3905 & 0.1753 \\
-0.4365 & 0.2055 & 1.3115 & 0.0016 & 0.1284 & -0.3973 \\
0.0163 & 0.3753 & 0.2328 & 0.7454 & 0.2239 & 0.0174 \\
0.1652 & -0.3924 & -0.3188 & 0.2085 & 0.7636 & 0.1482 \\
-0.8850 & 0.3298 & 0.5763 & 0.0609 & 0.2084 & 0.1941
\end{array}\right] .
$$

Then, the solutions computed by the Algorithm coincide with those drawn in Figure 1.

\subsection{A solution of Step 8 in the Algorithm 2}

The goal of this subsection is to provide a method to construct a nontrivial idempotent matrix $T$ satisfying $\Sigma K T=T \Sigma K$.

Proposition 11. Consider the matrices $\Sigma$ and $K$ of decomposition (10) of the matrix $A_{2} \in \mathbb{R}^{n \times n}$. There exists a nontrivial idempotent matrix $T$ such that $\Sigma K T=T \Sigma K$ if and only if there exists a nonsingular matrix $S \in \mathbb{R}^{r \times r}$ such that

$$
\Sigma K=S\left[\begin{array}{cc}
S_{1} & O \\
O & S_{2}
\end{array}\right] S^{-1},
$$

with $S_{1} \in \mathbb{R}^{a \times a}, S_{2} \in \mathbb{R}^{(r-a) \times(r-a)}$ nonsingular matrices, and $1 \leq a<r$.

Proof. Assume that $T$ is a nontrivial idempotent matrix, that is $O \neq T \neq I_{r}$. It is well known that there exists a nonsingular matrix $S \in \mathbb{R}^{r \times r}$ such that

$$
T=S\left[\begin{array}{ll}
I_{a} & O \\
O & O
\end{array}\right] S^{-1}
$$

with $1 \leq a<r$. We partition

$$
\Sigma K=S\left[\begin{array}{ll}
S_{1} & S_{3} \\
S_{4} & S_{2}
\end{array}\right] S^{-1}
$$

according to the size of the blocks of $S^{-1} T S$. From $\Sigma K T=T \Sigma K$ we get $S_{3}=O$ and $S_{4}=O$. The nonsingularity of $S_{1}$ and $S_{2}$ follows from the nonsingularity of $\Sigma K$.

Conversely, assuming that $\Sigma K$ is partitioned as in 15 and setting $T$ as in (16), it is easy to see that $T$ is idempotent and commute with $\Sigma K$. 
We observe that such a matrix $T$ can always be constructed since $\Sigma K$ can always be partitioned as in (15) by using its Jordan canonical form. In particular, when $\Sigma K$ is diagonalizable, this issue can easily be done. Even more, in this case, we can construct several matrices $T$ with different rank by choosing adequate blocks in $\Sigma K$. An alternative approach to select such a matrix $T$ is to use the Schur decomposition of $\Sigma K$ provided that it yields to a block diagonal matrix.

\section{A new approach to compute the projector}

In this section, we are going to give a new way of calculating the projector matrix that relates the solutions of two autonomous systems ordered under the sharp partial order. In order to do that we consider the following result by D.S. Rakiĉ, D.S. Djordjević [18, Theorem 2.6].

Proposition 12. Let $A_{1}, A_{2} \in \mathbb{R}^{n \times n}$ be matrices having index 1. Then, the following assertions are equivalent

(a) $A_{1} \stackrel{\#}{\leq} A_{2}$.

(b) There exists an idempotent matrix $Q \in \mathbb{R}^{n \times n}$ that commutes with $A_{2}$ and satisfies $A_{1}=Q A_{2}$.

Next, we give the following result where the link between the solutions of two ordered systems is specified.

Theorem 13. Let $A_{1}, A_{2} \in \mathbb{R}^{n \times n}$ be the state matrices of two autonomous systems given by (3) ordered under the sharp partial order. Then, there exists an idempotent matrix $Q \in \mathbb{R}^{n \times n}$ such that the solutions of both systems are related by

$$
x(k)=Q \bar{x}(k)
$$

provided that both initial conditions are equal, that is $\bar{x}(0)=x(0)$.

Proof. Assume that $A_{1} \stackrel{\#}{\leq} A_{2}$. By Proposition 12 , there exists an idempotent matrix $Q \in \mathbb{R}^{n \times n}$ such that $A_{1}=Q A_{2}=A_{2} Q$. Since $Q^{k}=Q$ for any positive integer $k$ and from $\bar{x}(k)=A_{2}^{k} x(0)$, we get

$$
x(k)=A_{1}^{k} x(0)=\left(Q A_{2}\right)^{k} x(0)=Q^{k} A_{2}^{k} x(0)=Q \bar{x}(k) .
$$

Assume that $A_{1} \stackrel{\#}{\leq} A_{2}$. From Proposition 1

$$
A_{1}=P\left[\begin{array}{ccc}
C & & \\
& O & \\
& & O
\end{array}\right] P^{-1} \text { and } A_{2}=P\left[\begin{array}{ccc}
C & & \\
& Y & \\
& & O
\end{array}\right] P^{-1},
$$


for nonsingular matrices $C \in \mathbb{R}^{r \times r}$ and $Y \in \mathbb{R}^{\ell \times \ell}$. By Theorem $12, A_{1}=$ $Q A_{2}=A_{2} Q$ for some idempotent matrix $Q$. Now, $Q A_{1}=Q A_{2}=A_{1}$. Similarly, $A_{1} Q=A_{1}$. Then, from

$$
P\left[\begin{array}{ccc}
C & & \\
& O & \\
& & O
\end{array}\right] P^{-1}=Q P\left[\begin{array}{ccc}
C & & \\
& O & \\
& & O
\end{array}\right] P^{-1} .
$$

and denoting

$$
P^{-1} Q P=\left[\begin{array}{c|cc}
M & N & T \\
\hline R & W \\
V & W
\end{array}\right]
$$

we have $M=I_{r}, R=O$, and $V=O$. Similarly, from $A_{1}=A_{1} Q$ we get $N=O$, $T=O$. Since $Q$ is idempotent, it then follows $W^{2}=W$. Now, using that $A_{1}=Q A_{2}=A_{2} Q$ we arrive at

$$
W\left[\begin{array}{ll}
Y & O \\
O & O
\end{array}\right]=O \quad \text { and } \quad\left[\begin{array}{cc}
Y & O \\
O & O
\end{array}\right] W=O
$$

The nonsingularity of $Y$ leads to $W=\left[\begin{array}{cc}O & \\ & Z\end{array}\right]$. Hence, we have obtained the following result, which gives the set of all the idempotent matrices appearing in Proposition 12 .

Lemma 14. Let $A_{1}, A_{2} \in \mathbb{R}^{n \times n}$ be matrices having index 1 such that $A_{1} \stackrel{\#}{\leq} A_{2}$ and written as in (4) and (5). Then the set of all idempotent matrices $Q_{Z} \in$ $\mathbb{R}^{n \times n}$ such that $A_{1}=Q_{Z} A_{2}=A_{2} Q_{Z}$ is given by

$$
Q_{Z}=P\left[\begin{array}{ccc}
I_{r} & & \\
& O & \\
& & Z
\end{array}\right] P^{-1}
$$

where $Z \in \mathbb{R}^{(n-r-\ell) \times(n-r-\ell)}$ satisfies $Z^{2}=Z$.

Notice that the particular case $Z=O$ leads to the easiest situation $Q_{O}=$ $A_{1} A_{1}^{\#}=A_{1}^{\#} A_{1}$.

Remark 5. For two systems (given by the matrices $A_{1}$ and $A_{2}$ ) ordered under the sharp partial order, we highlight that the solutions are independent of the choice of $Z$ since $A_{1}=Q_{Z} A_{2}=A_{2} Q_{Z}$.

Example 15. Consider the matrices $A_{1}, A_{2}$, and $P$ given in Example 4. By Lemma 14, the solutions of the autonomous systems given by (3) can be obtained 
by the idempotent matrix $Q_{O}$, computed as

$$
\begin{aligned}
Q_{O}= & P\left[\begin{array}{lll}
I_{2} & & \\
& O_{2} & \\
& & O_{2}
\end{array}\right] P^{-1} \\
& =\left[\begin{array}{rrrrrr}
0.2915 & -0.0739 & -0.1214 & 0.1612 & 0.0886 & -0.0573 \\
0.5174 & 0.1507 & -0.3213 & 0.6237 & 0.0415 & -0.0021 \\
-1.6856 & 0.6995 & 0.6000 & -0.6059 & -0.6236 & 0.4272 \\
-0.4404 & 0.6480 & -0.0178 & 0.3990 & -0.3538 & 0.2759 \\
0.4596 & -0.2859 & -0.1279 & 0.0512 & 0.2091 & -0.1501 \\
-1.2284 & 0.6181 & 0.3966 & -0.3117 & -0.4990 & 0.3496
\end{array}\right]
\end{aligned}
$$

by means of the relation

$$
\left[\begin{array}{l}
x_{1}(k) \\
x_{2}(k) \\
x_{3}(k) \\
x_{4}(k) \\
x_{5}(k) \\
x_{6}(k)
\end{array}\right]=Q_{O}\left[\begin{array}{l}
\bar{x}_{1}(k) \\
\bar{x}_{2}(k) \\
\bar{x}_{3}(k) \\
\bar{x}_{4}(k) \\
\bar{x}_{5}(k) \\
\bar{x}_{6}(k)
\end{array}\right]
$$

and their trajectories are shown in Figure 1.

\section{Conclusions}

This paper presents a new technique to tackle problems in two areas: Matrix Partial Orders and Autonomous Linear Systems. Specifically, we have introduced the idea of ordered autonomous systems under the sharp partial order and we have compared the solutions of two ordered systems obtaining upper bounds of the gap between them. This comparison allows us to see the relationship between the solutions from different points of view: perturbations, projections.

\section{Acknowledgements}

We thank the Referees for their valuable comments that helped us to improve considerably this paper.

This research was partially supported by Ministerio de Economía, Industria y Competitividad of Spain (Red de Excelencia MTM2017-90682-REDT).

\section{References}

[1] J. Angulo. Mathematical morphology for matrix-valued images, Conference on Matrix Information Gemotries, Palaiseau, France, 2011.

[2] R.B. Bapat. Linear Algebra and Linear Models. Third Edition, Springer, New York, 2012. 
[3] A. Ben-Israel, T.N.E. Greville. Generalized Inverses: Theory and Applications. Wiley and Sons, New York, 1974.

[4] S.L. Campbell, C.D. Meyer, Generalized Inverses of Linear transformations, SIAM, Philadelphia, 2009.

[5] C. Coll, A. Herrero, E. Sánchez, N. Thome. Partially ordered structured systems (in Spanish), VI MACI 2017, Matemática Aplicada, Computacional e Industrial, Argentina, 2017.

[6] J. Cronin. Ordinary Differential Equations: Introduction and Qualitative Theory, Third Edition, Chapman \& Hall/CRC, New York, 2008.

[7] D.S. Cvetković-Ilić, D. Mosić, Y. Wei. Partial orders on $\mathcal{B}(H)$, Linear Algebra and its Applications, 481, 115-130 (2015).

[8] P.A. Gagniuc. Markov chains: From theory to implementation and experimentation. Wiley and Sons, New York, 2017.

[9] R.A. Horn, C.R. Johnson. Matrix analysis, Second Edition, Cambridge, New York, 2013.

[10] R.E. Hartwig, K. Spindelböck. Matrices for which $A^{*}$ and $A^{\dagger}$ commute. Linear and Multilinear Algebra, 14, 241-256 (1984).

[11] M. Kot. Elements of Mathematical Ecology, Cambridge University Press, 2001.

[12] A.J. Lotka. Elements of mathematical biology, Dover Publications Inc., New York, 1956.

[13] O. Makarenkov, P. Nistri. Periodic solutions for planar autonomous systems with nonsmooth periodic perturbations. Journal of Mathematical Analysis and Applications, 338, 1401-1417 (2008).

[14] S. Malik, L. Rueda, N. Thome. Further properties on the core partial order and other matrix partial orders Linear and Multilinear Algebra, 62, 12, 1629-1648 (2014).

[15] D.R. Merkin. Stability of linear autonomous systems, Springer, New York, 1997.

[16] D. Mosić, D.S. Cvetković-Ilić. Some orders for operators on Hilbert spaces, Applied Mathematics and Computation, 275, 229-237 (2016).

[17] S.K. Mitra, P. Bhimasankaram, S.B. Malik. Matrix Partial Orders, Shorted Operators and Applications. World Scientific Publishing Company, London, 2010 .

[18] D.S. Rakiĉ, D.S. Djordjević. Star, sharp, core and dual core partial order in rings with involution, Applied Mathematics and Computation, 259, 800818 (2015). 
[19] G.P.H. Styan. Notes on the distribution of quadratic forms in singular normal variables, Biometrika, 57, 567-572 (1970).

[20] J.Y. Vélez-Cerrada, J. Robles, N. Castro-González. Error bounds for the perturbation of the Drazin inverse under some geometrical conditions, Applied Mathematics and Computation, 215, 2154-2161 (2009).

[21] Y. Wei. Perturbation bound of the Drazin inverse, Applied Mathematics and Computation, 125, 2-3, 231-244 (2002). 


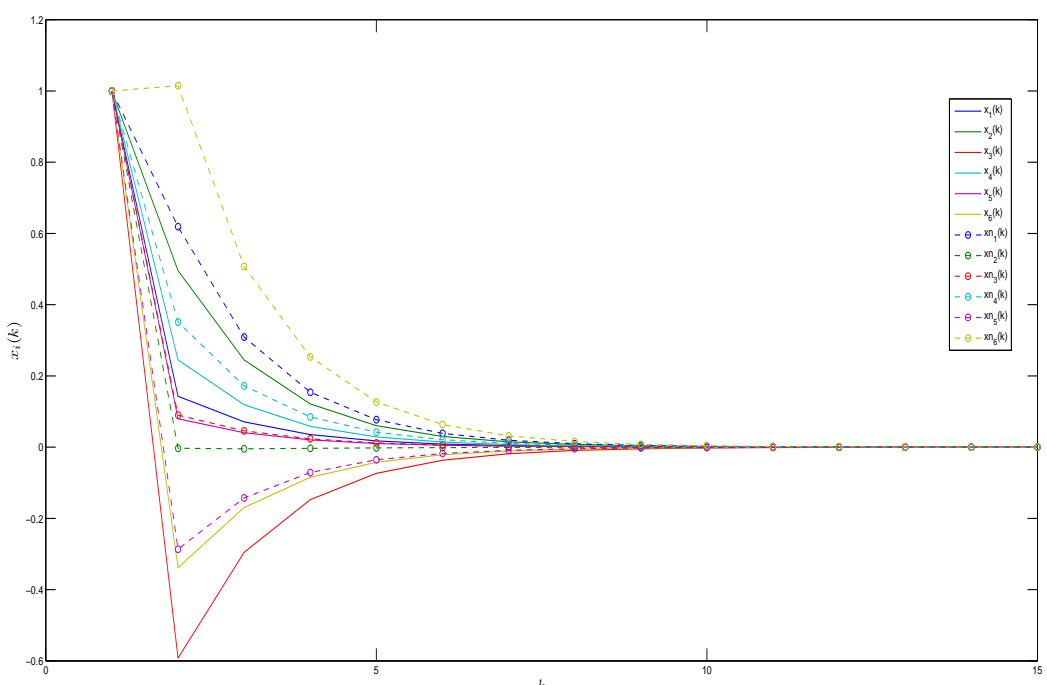

Figure 1: Evolution of the 15th first iterations of the solutions $x_{i}(k)$ and $\bar{x}_{i}(k)$ 


\begin{tabular}{|c|c|c|c|c|}
\hline$A_{2}$ & $\rho\left(A_{2}\right)$ & $\begin{array}{c}\text { Asymptotic } \\
\text { stability }\end{array}$ & $\begin{array}{c}\text { Singular } \\
\text { values }\end{array}$ & $\|\Sigma\|_{F}^{k} \underset{k \rightarrow \infty}{\longrightarrow} 0$ \\
\hline \hline Example 9 & $<1$ & yes & $\sigma_{i}<1, \forall i$ & yes \\
\hline$\left[\begin{array}{cc}0.8071 & 0.8071 \\
0 & 0\end{array}\right]$ & $<1$ & yes & $\begin{array}{c}\exists i: \sigma_{i}<1, \\
\exists j \neq i: \sigma_{j} \geq 1\end{array}$ & yes/no \\
\hline Example 10 & $<1$ & yes & $\exists i: \sigma_{i} \geq 1$ & no \\
\hline$\nexists$ & $\geq 1$ & - & $\sigma_{i}<1, \forall i$ & - \\
\hline$\left[\begin{array}{ll}0 & 0 \\
2 & 2\end{array}\right]$ & $\geq 1$ & no & $\begin{array}{c}\exists i: \sigma_{i}<1, \\
\exists j \neq i: \sigma_{j} \geq 1\end{array}$ & yes/no \\
\hline $\operatorname{diag}(2,1,0)$ & $\geq 1$ & no & $\exists i: \sigma_{i} \geq 1$ & no \\
\hline
\end{tabular}

Table 1: Relationship between eigenvalues and singular values of the matrix $A_{2}$ to understand the upper bound in 14. 


\begin{tabular}{|c|c|c|}
\hline$k$ & $\left\|x_{k}-\bar{x}_{k}\right\|$ & $\|T-I\|_{F}\|\Sigma\|_{F}^{k}\|x(0)\|$ \\
\hline \hline 5 & 0.0626958 & 0.0876378 \\
10 & 0.00188192 & 1.11167 \\
15 & 0.00005876 & 0.476665 \\
20 & $1.83613 \times 10^{-6}$ & 0.204387 \\
25 & $5.73668 \times 10^{-8}$ & 0.0876378 \\
100 & $1.51336 \times 10^{-30}$ & $2.66857 \times 10^{-7}$ \\
200 & $1.18846 \times 10^{-60}$ & $1.17777 \times 10^{-14}$ \\
\hline
\end{tabular}

Table 2: Difference between the solutions of both systems and the upper bound. 